\title{
Global and Sensitivity Analyses of Unconcerned COVID-19 Cases in Nigeria: A Mathematical Modeling Approach
}

\author{
FANIRAN, T. S. ${ }^{1}$, BAKARE, E. A. ${ }^{2,3}$, POTUCEK, R. ${ }^{* 4}$, AYOOLA, E. O. ${ }^{5}$ \\ ${ }^{1}$ Department of Computer Science, Lead City University, \\ Toll Gate Area, Ibadan, Oyo State, NIGERIA \\ ${ }^{2}$ Disease Modelling, Simulation and Data Science Network, Africa (MODSIMID.NET, Africa) \\ ${ }^{3}$ Department of Mathematics, Federal University Oye Ekiti, \\ Oye-Are Road, Ekiti State, NIGERIA \\ ${ }^{4}$ Department of Mathematics and Physics, University of Defence, \\ Kounicova 65, 66210 Brno, CZECH REPUBLIC \\ ${ }^{5}$ Department of Mathematics, University of Ibadan, \\ Oduduwa Road, Ibadan, Oyo State, NIGERIA
}

\begin{abstract}
Covid-19 is caused by severe acute respiratory syndrome coronavirus 2 (SARS-CoV-2). Many measures have been made by World Health Organization (WHO), but these may be threatened by unconcerned infectious individuals (some infectious individuals who do not take the disease serious, by ignoring non-pharmaceutical intervention). A system of nonlinear ordinary differential equations that absorbs a class of unconcerned infectious individuals, is developed. An invasion threshold parameter, $R_{c}$, is derived using the next generation matrix approach. This is used to establish the global stability of COVID-19-free equilibrium points. The global asymptotic stability of COVID-19 persistence equilibrium solution is studied through the use of suitable LaSalle's Invariance Principle with a Lyapunov function of Goh-Volterra type. The intervention of the model key parameters is assessed through sensitivity analysis. Our results indicate that increase in the rate of hospitalization of the asymptomatic infectious and unconcerned infectious individuals after a compulsory national testing, could bring $R_{c}$ below one. Our results suggest that there should be compulsory national testing and continuous enhancement, the awareness through effective risk communication concerning COVID-19 to the general public. Numerical simulations are carried out to validate the analytical results.
\end{abstract}

Key-Words: COVID-19, unconcerned infectious individuals, testing, stability analyses, sensitivity analysis

Received: January 11, 2021. Revised: April 22, 2021. Accepted: May 5, 2021. Published: May 28, 2021.

\section{Introduction}

The novel coronavirus, COVID-19, started in mainland China, with a geographical emphasis at Wuhan, the capital city of Hubei province [1] and has widely spread globally. Previous outbreaks to COVID19 were the Severe Acute Respiratory Syndrome (SARS-CoV), reported in Asia in February 2003, resulting in 8422 cases with a case fatality rate of $11 \%$ [2]. Later, in 2012, the Middle East Respiratory Syndrome (MERS-CoV) was identified in Saudi Arabia and infected 2506 people, killing 862 between years 2012 and 2020 [2].

The established clinical symptoms of COVID19 include fever, cough, shortness of breath, sore throat, loss of taste and smell [3]. Currently, several countries have developed the vaccine for COVID-19 which does not guarantee total safety from the disease being transmitted. Therefore, individual behaviour, i.e. social distancing, early self-isolation, as well as preventive measures, such as hand washing, covering when coughing are critical to controlling the spread of the disease [4]. Additionally to these measures, several restrictions and quarantine of the exposed individuals, are necessary.

Many authors have developed different models to study the dynamics of COVID-19, see for instance, [5], [6], [7], [8], [9], [10], [11], [12], [15], [16]. Also, Bian et al. [5] developed a mathematical model for COVID-19 by focusing on the practical implications of public health interventions and measures. Their model also incorporates an asymptomatic group. They carried out a sensitivity analysis of the model which shows that interventions, such as intensive contact tracing, followed by quarantine 
and isolation, can effectively reduce the basic reproduction number and transmission risk, with the effect of travel restrictions adopted by Wuhan on 2019$\mathrm{nCoV}$ infection in Beijing being almost equivalent to increasing quarantine by a 1000 baseline value.

Tridip et al. [10] formulated a model on the COVID-19 transmission that incorporates lock-down effect and variability in transmission between symptomatic and asymptomatic populations with the former being a fast spreader of the disease. Three states, namely, Maharashtra, Delhi and Telanguana, were used as case studies, to assess the effect of the current 21 days lock-down in terms of reduction cases and deaths. Their results suggest that 21 days lockdown will have no impact in Maharashtra and overall India. Their results further suggest that the presence of a higher percentage of COVID-19 super spreader will further deteriorate the situation in Maharashtra.

Nkamba et al [11] predicted the COVID-19 epidemic in Cameroon using early reported case data. They predicted the impact of containment measures and the impact of undetected people on the epidemic trend and characteristics of COVID-19. They computed the basic reproduction which is used to establish the global stability of disease-free equilibrium points. Also, they demonstrated the global stability of the endemic equilibrium solution using the Lyapunov function. They carried out global sensitivity analysis using Latin Hypercube Sampling (LHS), to know the parameters that strongly influence the dynamics of COVID-19 infection.

Enahoro et al. [17] made use of a mathematical model to assess the impact of an imperfect antiCOVID-19 vaccine on the control of COVID-19 in the United State. Theoretical analysis of the model was done, as well as the model fitting and parameter estimation. They performed numerical simulations of the model, using baseline parameter values obtained from fitting the model with COVID-19 mortality data for the U.S. Their result indicated that at least, $82 \%$ of the susceptible U.S population need to be vaccinated with an assumed protective efficacy of $80 \%$ for an anti-COVID-19 vaccine. They concluded that the imperfect vaccine will be greatly enhanced if the vaccination program is combined with other interventions, like social distancing, wearing face masks, etc.

In the current complex and geopolitical situation adversely affected by the COVID-19 pandemic, the importance of human resource management is growing (see [18]), especially in the health sector and in the security forces. In connection with the management of local crisis situations, psychological support for doctors, nurses, paramedics and all medical staff is gaining in importance (see [19]).

At the same time, the demands on the training of security specialists dealing with crisis management are growing, due to the needs and rapidly changing requirements for the necessary measures in society due to the current epidemiological situation (see [20]).

In a society affected by the COVID-19 pandemic, it is necessary to take a number of preventive measures aimed at increasing the level of preparedness of the intervening teams and components of the integrated rescue system (see [21]). In particular, adequate mechanisms and procedures need to be put in place as a matter of urgency and flexibility in the activities provided by the emergency medical service, but also by first-line doctors. These procedures - especially in testing, anti-COVID-19 vaccination, but also in tracing - need to minimize the negative effects of a pandemic on human health.

The individual waves of the COVID-19 pandemic can be modeled, for example, using the threat life cycle and its various phases (see [22]). There are a number of epidemic models. Some relevant studies can be found in [13] and [14].

Olaniyi et al. [15] formulated an epidemic model by considering the transmission routes from symptomatic, asymptomatic, and hospitalized individuals. Their model was fitted to the corresponding cumulative number of hospitalized individuals reported by the Nigeria Centre for Disease Control (NCDC), using the Least Squares Method. They computed the basic reproduction number and constructed the Lyapunov function to investigate the stability of the model around a disease-free equilibrium point. They further made use of Pontryagin's Maximum Principle to analyze the optimal control of the model. They carried out numerical simulations to justify their theoretical work and their result revealed that the basic reproduction number can be brought to a value less than one in Nigeria if the current effective transmission rate of the disease can be reduced by $50 \%$.

Enahoro et al. [12] developed and analyzed a mathematical model of COVID-19 in Nigeria. They carried out a rigorous analysis of their model, by establishing locally-asymptotically stability equilibria. They parameterized the model using COVID-19 data published by Nigeria Centre for Disease Control (NCDC). This was used to assess the communitywide impact of various control and mitigation strategies in the entire Nigerian nation, as well as in two states (Kano and Lagos) within the Nigerian federation and the Federal Capital Territory (FCT), Abuja. Their result predicted that Nigeria would have recorded a devastatingly high COVID-19 mortality by April 2021 (in hundreds of thousands), in places where social-distancing, lockdown, and other community transmission reduction measures are not implemented.

Adeniyi et al. [16] proposed and analyzed a nonlinear mathematical model to investigate the effect of 
healthy sanitation and awareness on the transmission dynamics of coronavirus disease (COVID-19) prevalence in Nigeria. They carried out rigorous stability of the model equilibrium points to ascertain the basic reproduction number. They performed numerical simulations using real-life data to support the analytic results. Their results revealed that propagation of information on good hygiene over time induces behavioral change in individuals leading to a significant reduction in the number of quarantined and infectious individuals.

None of the above-mentioned models take into account the unconcerned infectious individuals compartment. In our effort to show novelty, we present a mathematical model of COVID-19 which investigates the contribution of unconcerned COVID19 cases in the occurrence of the second wave of COVID-19 in Nigeria.

Moreover, hospitalization is considered to be effective in reducing the transmission, mortality, and morbidity of COVID-19 and this is also incorporated. Thus, we formulate a mathematical model that captures this important compartment of unconcerned COVID-19 cases in Nigeria.

We compute the basic reproduction number, $R_{c}$, and investigate the existence and stability of COVID19-free and COVID-19 persistence equilibria. We prove that the disease-free equilibrium is globally asymptotically stable if the basic reproduction number $R_{c}$ is less than one.

The global asymptotic stability of endemic equilibrium solution is studied through the use of suitable LaSalle's Invariance Principle with a Lyapunov function of Goh-Volterra type [23]. Furthermore, we investigate the sensitivity indices of the basic reproduction number to the parameters of the formulated model, intending to know the contributory effects of the parameters in the transmission and spread of COVID-19 in the population. An interesting approach to problems connected with COVID-19 pandemic can be found also e.g. in [24] - [26].

Based on the above, we in this research focuses on the following: (i) To investigate the role of unconcerned infectious individuals on transmission dynamics of COVID-19; (ii) To carry out the stability analysis of COVID-19 free and COVID-19 endemic equilibrium solution, globally; (iii) To perform sophisticated sensitivity analysis of the basic reproduction number of COVID-19; while the research significance is as follows: (i) The model will help the public health authorities, government and policy makers to understand to what rate is the class of unconcerned infectious individuals influencing COVID-19 transmission and also intervene by organizing educational campaign program, on the risk of not adhering to nonpharmaceutical measures; (ii) The theoretical results will provide important insights into the disease behaviour and control. This will give insights whether the disease dies out of the population or persists in the population in the advancement of time; (iii) This study will give the policy makers, information on the most influential parameters that call for immediate intervention strategies, in order to halt the spread of the disease in the population.

This paper is organized as follows; we present a covid-19 unconcerned transmission model formulation and analysis of the reproduction number in Section 2. In Section 3, global stability analyses were discussed. In Section 4, the sensitivity analysis is presented while in section 5 , numerical simulations were discussed. In Section 6, we discuss the main conclusion and recommendations.

\section{Seven-Compartmental COVID-19 Model}

The total population size, $N_{H}$, is divided into seven stages; the susceptible population $S_{H}$, the exposed population $E_{H}$, the asymptomatic infectious population $A_{H}$, the unconcerned infectious population $I_{U}$, the concerned infectious population $I_{C}$, the hospitalized infectious population $H_{I}$ and the recovered infectious population $R_{H}$.

The population $S_{H}$ starts with a recruitment rate $\varepsilon$ (new births, immigration and emigration) and natural death of susceptible individuals with a rate $\mu_{h}$. Interaction between a susceptible and asymptomatic individuals or unconcerned infectious individuals or concerned infectious individuals are reduced from susceptible class with rates $\alpha, \sigma$ and $\rho$.

It is assumed that COVID-19 outbreaks have lasted for at least ten months and during this period of time, there might be new births or inflow of susceptible individuals from other places as well as natural deaths, which allow a demographic process to take place. The modification parameter $0<\psi<1$ accounts for the assumed reduction in COVID-19 transmission of concerned infectious individuals.

A fraction $\theta$ of the exposed individuals is asymptomatic. A fraction $\omega(0 \leq \omega \leq 1)$ of the remaining fraction $(1-\theta)$ of the exposed individuals are unconcerned infectious individuals, while a fraction $(1-\omega)$ of the remaining fraction, $1-\theta$, of the exposed individuals are concerned infectious individuals.

It is assumed that asymptomatic infected individuals show no symptoms while the unconcerned and concerned infectious individuals show mild to moderate symptoms of COVID-19 and hence they can still move around. It is assumed that the unconcerned infectious individuals believe they are showing symptoms of just common cold and catarrh thereby not taking COVID-19 non-pharmaceutical intervention seri- 
ous.

After screening, the asymptomatic infected individuals, the unconcerned and the concerned infectious individuals are hospitalized at rates $\kappa_{1}, \kappa_{2}$ and $\kappa_{3}$. COVID-19 caused-death occurs in unconcerned infectious, concerned infectious and hospitalized individuals at rates $\delta_{1}, \delta_{2}$ and $\delta_{3}$. There is also recovery of $A_{H}, I_{U}, I_{C}$ and $H_{I}$ at the rates $\tau_{1}, \tau_{2}, \tau_{3}$ and $\tau_{4}$.

The summary of these parameters together with their values is stated in the following Table 1:
Table 1: Summary of the parameters

\begin{tabular}{|c|c|c|c|}
\hline Parameter & Meaning & Value & Reference \\
\hline$\beta$ & $\begin{array}{l}\text { Incubation period } \\
\text { for COVID-19 }\end{array}$ & 0.142 & [27] \\
\hline$\alpha$ & $\begin{array}{l}\text { Contact rate between } \\
\text { susceptible and } \\
\text { asymptomatic individual }\end{array}$ & 0.001 & [11] \\
\hline$\rho$ & $\begin{array}{l}\text { Contact rate between } \\
\text { susceptible and } \\
\text { concerned infectious }\end{array}$ & 0.001 & [11] \\
\hline$\sigma$ & $\begin{array}{l}\text { Contact rate between } \\
\text { susceptible and } \\
\text { unconcerned infectious }\end{array}$ & 0.001 & [11] \\
\hline$\theta$ & $\begin{array}{l}\text { Fraction of the exposed } \\
\text { individuals who } \\
\text { are asymptomatic }\end{array}$ & 0.8262 & [11] \\
\hline$\omega$ & $\begin{array}{l}\text { Fraction of the exposed } \\
\text { individuals who are } \\
\text { unconcerned infectious }\end{array}$ & $0.8262(0,1)$ & Assumed \\
\hline$\tau_{1}, \tau_{2}$ & Recovery rates & & \\
\hline $\begin{array}{l}\tau_{3}, \tau_{4} \\
\varepsilon\end{array}$ & Recruitment rate & $\begin{array}{l}\frac{1}{15} \\
3000\end{array}$ & $\begin{array}{l}\text { [28] } \\
\text { Assumed }\end{array}$ \\
\hline$\mu_{h}$ & $\begin{array}{l}\text { Natural death rate } \\
\text { of individuals }\end{array}$ & 0.06 & [11] \\
\hline $\begin{array}{l}\delta_{1}, \delta_{2} \\
\delta_{3} \\
\kappa_{1}, \kappa_{2}\end{array}$ & $\begin{array}{l}\text { COVID-19-caused } \\
\text { death rates } \\
\text { Hospitalization rates }\end{array}$ & $(0.02-0.1)$ & [28] \\
\hline & & $(0-1)$ & [10] \\
\hline$\psi$ & $\begin{array}{l}\text { Reduction factor in } \\
\text { COVID-19 transmission } \\
\text { for concerned infectious }\end{array}$ & $(0-1)$ & [10] \\
\hline
\end{tabular}

Arising from the above description, the mathemat- ical model is arrived at:

$$
\begin{aligned}
\frac{\mathrm{d} S_{H}}{\mathrm{~d} t} & =\varepsilon-\alpha S_{H} A_{H}-\sigma S_{H} I_{U} \\
& -\rho \psi S_{H} I_{C}-\mu_{h} S_{H}, \\
\frac{\mathrm{d} E_{H}}{\mathrm{~d} t} & =\alpha S_{H} A_{H}+\sigma S_{H} I_{U} \\
& +\rho \psi S_{H} I_{C}-\left(\beta+\mu_{h}\right) E_{H}, \\
\frac{\mathrm{d} A_{H}}{\mathrm{~d} t} & =\theta \beta E_{H}-\left(\kappa_{1}+\tau_{1}+\mu_{h}\right) A_{H}, \\
\frac{\mathrm{d} I_{U}}{\mathrm{~d} t} & =\omega(1-\theta) \beta E_{H} \\
& -\left(\kappa_{2}+\delta_{1}+\tau_{2}+\mu_{h}\right) I_{U}, \\
\frac{\mathrm{d} I_{C}}{\mathrm{~d} t} & =(1-\omega)(1-\theta) \beta E_{H} \\
& -\left(\kappa_{3}+\delta_{2}+\tau_{3}+\mu_{h}\right) I_{C},
\end{aligned}
$$

$$
\begin{aligned}
\frac{\mathrm{d} H_{I}}{\mathrm{~d} t} & =\kappa_{1} A_{H}+\kappa_{2} I_{U}+\kappa_{3} I_{C} \\
& -\left(\delta_{3}+\tau_{4}+\mu_{h}\right) H_{I}, \\
\frac{\mathrm{d} R_{H}}{\mathrm{~d} t} & =\tau_{1} A_{H}+\tau_{2} I_{U}+\tau_{3} I_{C} \\
& +\tau_{4} H_{I}-\mu_{h} R_{H} .
\end{aligned}
$$

with initial conditions

$$
\begin{array}{r}
S_{H}(0)=S_{h}^{o}>0, \quad E_{H}(0)=E_{H}^{o}, \\
A_{H}(0)=A_{H}^{o}(0)>0 \\
I_{U}(0)=I_{U}^{o}(0)>0, \quad I_{C}(0)=I_{C}^{o}(0)>0 \\
H_{I}(0)=H_{I}^{o}(0), \quad R_{H}(0)=R_{H}^{o}(0)>0
\end{array}
$$

where the model parameters are non negative. For biological reasons, the model is analysed in the feasible region

$$
\begin{gathered}
D=\left(\left(S_{H}, E_{H}, A_{H}, I_{U}, I_{C}, H_{I}, R_{H}\right) \in \mathbb{R}_{+}^{7}:\right. \\
\left.0 \leq S_{H} \leq \frac{\varepsilon}{\mu_{h}}, N_{H} \leq \frac{\varepsilon}{\mu_{h}}\right)
\end{gathered}
$$

that can be shown to be positively invariant with respect to the system (11) - (7). Thus, the model is well posed mathematically and epidemiologically in $D$.

The first five equations, i.e. (11) - (5), are independent of the compartments $H_{I}$ and $R_{H}$, i.e. (6) - (7). Therefore, after decoupling the equations for $H_{I}$ and $R_{H}$ from model (1) - (7), analysis is concentrated on 
the remaining equations of (11) - (7) which becomes

$$
\begin{aligned}
\frac{\mathrm{d} S_{H}}{\mathrm{~d} t} & =\varepsilon-\alpha S_{H} A_{H}-\sigma S_{H} I_{U} \\
& -\rho \psi S_{H} I_{C}-\mu_{h} S_{H}, \\
\frac{\mathrm{d} E_{H}}{\mathrm{~d} t} & =\alpha S_{H} A_{H}+\sigma S_{H} I_{U} \\
& +\rho \psi S_{H} I_{C}-\left(\beta+\mu_{h}\right) E_{H}, \\
\frac{\mathrm{d} A_{H}}{\mathrm{~d} t} & =\theta \beta E_{H}-\left(\kappa_{1}+\tau_{1}+\mu_{h}\right) A_{H}, \\
\frac{\mathrm{d} I_{U}}{\mathrm{~d} t} & =\omega(1-\theta) \beta E_{H} \\
& -\left(\kappa_{2}+\delta_{1}+\tau_{2}+\mu_{h}\right) I_{U}, \\
\frac{\mathrm{d} I_{C}}{\mathrm{~d} t} & =(1-\omega)(1-\theta) \beta E_{H} \\
& -\left(\kappa_{3}+\delta_{2}+\tau_{3}+\mu_{h}\right) I_{C} .
\end{aligned}
$$

\subsection{Basic Reproduction Number}

To establish the global stability of disease-free equilibrium solution, it is required that the basic reproduction number $R_{c}$, is computed. This is done by expressing (11) - (7) as the difference between the rate of new infection in each infected compartment $F$ and the rate of transfer between each infected compartment $G$. Hence, we have

$$
\begin{aligned}
{\left[\begin{array}{c}
\frac{\mathrm{d} E_{H}}{\mathrm{~d} t} \\
\frac{\mathrm{d} A_{H}}{\mathrm{~d} t} \\
\frac{\mathrm{d} I_{U}}{\mathrm{~d} t} \\
\frac{\mathrm{d} I_{C}}{\mathrm{~d} t}
\end{array}\right] } & F-G \\
- & {\left[\begin{array}{c}
\alpha S_{H} A_{H}+\sigma S_{H} I_{U}+\rho \psi S_{H} I_{C} \\
0 \\
0 \\
0 \\
m_{1} E_{H} \\
-\theta \beta E_{H}+m_{2} A_{H} \\
-\omega(1-\theta) \beta E_{H}+m_{3} I_{U} \\
-(1-\omega)(1-\theta) \beta E_{H}+m_{4} I_{C}
\end{array}\right], \quad(13) }
\end{aligned}
$$

where

$$
\begin{aligned}
& m_{1}=\beta+\mu_{h}, \\
& m_{2}=\kappa_{1}+\tau_{1}+\mu_{h}, \\
& m_{3}=\kappa_{2}+\delta_{1}+\tau_{2}+\mu_{h}, \\
& m_{4}=\kappa_{3}+\delta_{2}+\tau_{3}+\mu_{h} .
\end{aligned}
$$

$R_{c}$ is given as

$$
R_{c}=\sqrt{\begin{array}{l}
\frac{S_{0} \beta \alpha \theta}{m_{1} m_{2}}+\frac{S_{0} \beta \sigma \omega(1-\theta)}{m_{1} m_{3}} \\
+\frac{S_{0} \beta \rho \psi(1-\omega)(1-\theta)}{m_{1} m_{4}}
\end{array}},
$$

so

$$
\begin{aligned}
R_{c}^{2} & =\frac{S_{0} \beta \alpha \theta}{m_{1} m_{2}}+\frac{S_{0} \beta \sigma \omega(1-\theta)}{m_{1} m_{3}} \\
& +\frac{S_{0} \beta \rho \psi(1-\omega)(1-\theta)}{m_{1} m_{4}}
\end{aligned}
$$

and

$$
R_{c}^{2}=R_{A_{H}}+R_{I_{U}}+R_{I_{C}} .
$$

Disease-free equilibrium of the model is given as

$$
E_{0}=\left(\frac{\varepsilon}{\mu_{h}}, 0,0,0,0\right) .
$$

It can also be written as

$$
E_{0}=\left(S_{0}, 0,0,0,0\right) .
$$

$R_{A_{H}}$ is the average number of secondary infections generated by an asymptomatic individual, $R_{I_{U}}$ is the average number of secondary infections generated by an unconcerned infectious individual and $R_{I_{C}}$ is the average number of secondary infections generated by a concerned infectious individual. When $\omega$ is near one, $R_{c}$ is largely affected by asymptomatic infectious and unconcerned infectious individuals.

\section{Global Stability Analysis}

We shall establish a global asymptotic stability of the disease-free equilibrium, that is solution trajectories of sub-population, irrespective of where they start, converge to the disease-free equilibrium solution for $R_{c}<1$. This is stated and proved below.

\subsection{Global Stability of COVID-19 Extinction Equilibrium Solution}

Theorem 3.1: The disease-free equilibrium solution is globally asymptotically stable if $R_{c}<1$ and unstable if $R_{c}>1$.

Proof: Consider the following linear Lyapunov function

$$
L=E_{H}+\frac{\alpha S_{0}}{m_{2}} A_{H}+\frac{\sigma S_{0}}{m_{3}} I_{U}+\frac{\rho \psi S_{0}}{m_{4}} I_{C} .
$$


Since

$$
\begin{aligned}
\frac{\mathrm{d} L}{\mathrm{~d} t} & =\alpha S_{H} A_{H}+\sigma S_{H} I_{U}+\rho \psi S_{H} I_{C} \\
& -m_{1} E_{H}+\frac{\alpha S_{0}}{m_{2}}\left(\theta \beta E_{H}-m_{2} A_{H}\right) \\
& +\frac{\sigma S_{0}}{m_{3}}\left(\omega(1-\theta) \beta E_{H}-m_{3} I_{U}\right) \\
& +\frac{\rho \psi S_{0}}{m_{4}}\left((1-\omega)(1-\theta) \beta E_{H}-m_{4} I_{C}\right),
\end{aligned}
$$

then

$$
\begin{aligned}
\frac{\mathrm{d} L}{\mathrm{~d} t} & =S_{H}\left(\alpha A_{H}+\sigma I_{U}+\rho \psi I_{C}\right) \\
& -S_{0}\left(\alpha A_{H}+\sigma I_{U}+\rho \psi I_{C}\right) \\
& +\left(\frac{S_{0} \beta \alpha \theta}{m_{1} m_{2}}+\frac{S_{0} \beta \sigma \omega(1-\theta)}{m_{1} m_{3}}\right. \\
& \left.+\frac{S_{0} \beta \rho \psi(1-\omega)(1-\theta)}{m_{1} m_{4}}-1\right) m_{1} E_{H},
\end{aligned}
$$

so

$$
\begin{aligned}
\frac{\mathrm{d} L}{\mathrm{~d} t} & =\left(S_{H}-S_{0}\right)\left(\alpha A_{H}+\sigma I_{U}+\rho \psi I_{C}\right) \\
& +\left(R_{c}^{2}-1\right) m_{1} E_{H} .
\end{aligned}
$$

Because $S_{H} \leq S_{0}$, we have

$$
\frac{\mathrm{d} L}{\mathrm{~d} t}=m_{1} E_{H}\left(R_{c}^{2}-1\right) \leq 0
$$

whenever $R_{c} \leq 1$.

Therefore

$$
\frac{\mathrm{d} L}{\mathrm{~d} t} \leq 0
$$

for $R_{c} \leq 1$ and

$$
\frac{\mathrm{d} L}{\mathrm{~d} t}=0
$$

if and only if $E_{H}=0, A_{H}=0, I_{U}=0, I_{C}=0$ or $S_{H}=S_{0}$ and $R_{c}=1$. Consequently, largest invariant set in

$$
\left\{\left(S_{H}, E_{H}, A_{H}, I_{U}, I_{C}, H_{I}, R_{H}\right) \in D ; \frac{\mathrm{d} L}{\mathrm{~d} t}=0\right\}
$$

is the singleton $E_{0}$ and by LaSalle's Invariance Principle [23] $E_{0}$ is globally asymptotically stable. The epidemiological implication of the above result is that COVID-19 can be eradicated from the population when $R_{c} \leq 1$ irrespective of the initial sizes of the sub-population of the model.

\subsection{Global Stability of COVID-19 Persistence Equilibrium Point}

The endemic equilibrium solution

$$
E_{1}=\left(S_{H}^{*}, E_{H}^{*}, A_{H}^{*}, I_{U}^{*}, I_{C}^{*}\right)
$$

satisfies the following equations:

$$
\begin{aligned}
& \varepsilon-\alpha S_{H}^{*} A_{H}^{*}-\sigma S_{H}^{*} I_{U}^{*} \\
& \quad-\rho \psi S_{H}^{*} I_{C}^{*}-\mu_{h} S_{H}^{*}=0 \\
& \alpha S_{H}^{*} A_{H}^{*}+\sigma S_{H}^{*} I_{U}^{*}+\rho \psi S_{H}^{*} I_{C}^{*}-m_{1} E_{H}^{*}=0 \\
& \quad \theta \beta E_{H}^{*}-m_{2} A_{H}^{*}=0 \\
& \quad \omega(1-\theta) \beta E_{H}^{*}-m_{3} I_{U}^{*}=0 \\
& \quad(1-\omega)(1-\theta) \beta E_{H}^{*}-m_{4} I_{C}^{*}=0
\end{aligned}
$$

Making $S_{H}^{*}, A_{H}^{*}, A_{U}^{*}$ and $I_{C}^{*}$ the subject of formula in (24) $-(28)$ gives

$$
\begin{aligned}
S_{H}^{*} & =\frac{\varepsilon}{\alpha A_{H}^{*}+\sigma I_{U}^{*}+\rho \psi I_{C}^{*}+\mu_{h}}, \\
A_{H}^{*} & =\frac{\theta \beta E_{H}^{*}}{m_{2}}, \\
I_{U}^{*} & =\frac{\omega(1-\theta) \beta E_{H}^{*}}{m_{3}}, \\
I_{C}^{*} & =\frac{(1-\omega)(1-\theta) \beta E_{H}^{*}}{m_{4}} .
\end{aligned}
$$

Adding (24) and (25), we get

$$
\varepsilon-\mu_{h} S_{H}^{*}-m_{1} E_{H}^{*}=0 .
$$

Substituting (29) $-(32)$ in (33) gives the following

$$
-\frac{m_{1} \mu_{h} R_{c}^{2}\left(E_{H}^{*}\right)^{2}}{\varepsilon}+\mu_{h}\left(R_{c}^{2}-1\right) E_{H}^{*}=0 .
$$

From (34), we can obtain $E_{H}^{*}$ to be

$$
E_{H}^{*}=\frac{\varepsilon}{m_{1}}\left(1-\frac{1}{R_{c}^{2}}\right) .
$$

Substituting $E_{H}^{*}$ in (30) - (33) gives

$$
\begin{aligned}
S_{H}^{*} & =\frac{\varepsilon}{\mu_{h} R_{c}^{2}}, \\
A_{H}^{*} & =\frac{\theta \beta \varepsilon}{m_{1} m_{2}}\left(1-\frac{1}{R_{c}^{2}}\right), \\
I_{U}^{*} & =\frac{\omega(1-\theta) \beta \varepsilon}{m_{1} m_{3}}\left(1-\frac{1}{R_{c}^{2}}\right), \\
I_{C}^{*} & =\frac{(1-\omega)(1-\theta) \beta \varepsilon}{m_{1} m_{4}}\left(1-\frac{1}{R_{c}^{2}}\right) .
\end{aligned}
$$


Thus, there is an existence of endemic equilibrium solution whenever $R_{c}>1$.

Furthermore, we establish the global asymptotic stability of the endemic equilibrium solution by proving that solution trajectories converge to the endemic equilibrium point for $R_{c}>1$. We shall carry out this, by constructing a suitable Lyapunov function of GohVolterra type, see [29]. The result below establishes the global stability stability of the endemic equilibrium solution $E_{1}$.

Theorem 3.2: The unique endemic equilibrium $E_{1}$, is globally asymptotically stable whenever $R_{c}>1$.

Proof: Given the following equations which are satisfied by the endemic equilibrium point $E_{1}$ :

$$
\begin{aligned}
\varepsilon=\left(\alpha A_{H}^{*}+\sigma I_{U}^{*}+\rho \psi I_{C}^{*}\right) S_{H}^{*} & +\mu_{h} S_{H}^{*}, \\
\left(\alpha A_{H}^{*}+\sigma I_{U}^{*}+\rho \psi I_{C}^{*}\right) S_{H}^{*} & =m_{1} E_{H}^{*}, \\
\theta \beta E_{H}^{*} & =m_{2} A_{H}^{*}, \\
(1-\omega)(1-\theta) \beta E_{H}^{*} & =m_{4} I_{C}^{*} .
\end{aligned}
$$

Consider the following Goh-Volterra Lyapunov function

$$
\begin{aligned}
V & =\left(S_{H}-S_{H}^{*}-S_{H}^{*} \ln \frac{S_{H}^{*}}{S_{H}}\right) \\
& +\left(E_{H}-E_{H}^{*}-E_{H}^{*} \ln \frac{E_{H}^{*}}{E_{H}}\right) \\
& +a\left(A_{H}-A_{H}^{*}-A_{H}^{*} \ln \frac{A_{H}}{A_{H}^{*}}\right) \\
& +b\left(I_{U}-I_{U}^{*}-I_{U}^{*} \ln \frac{I_{U}^{*}}{I_{U}}\right) \\
& +c\left(I_{C}-I_{C}^{*}-I_{C}^{*} \ln \frac{I_{C}}{I_{C}^{*}}\right)
\end{aligned}
$$

where

$$
a=\frac{\alpha S_{H}^{*}}{m_{2}}, \quad b=\frac{\sigma S_{H}^{*}}{m_{3}} \quad \text { and } \quad c=\frac{\rho \psi S_{H}^{*}}{m_{4}}
$$

Differentiating $V$ with respect to time gives

$$
\begin{aligned}
\frac{\mathrm{d} V}{\mathrm{~d} t} & =\left(1-\frac{S_{H}^{*}}{S_{H}}\right) S_{H}^{\prime}+\left(1-\frac{E_{H}^{*}}{E_{H}}\right) E_{H}^{\prime} \\
& +a\left(1-\frac{A_{H}^{*}}{A_{H}}\right) A_{H}^{\prime}+b\left(1-\frac{I_{U}^{*}}{I_{U}}\right) I_{U}^{\prime} \\
& +c\left(1-\frac{I_{C}^{*}}{I_{C}}\right) I_{C}^{\prime},
\end{aligned}
$$

SO

$$
\begin{aligned}
\frac{\mathrm{d} V}{\mathrm{~d} t} & =\left(1-\frac{S_{H}^{*}}{S_{H}}\right)\left(\left(\alpha A_{H}^{*}+\sigma I_{U}^{*}+\rho \psi I_{C}^{*}\right) S_{H}^{*}\right. \\
& \left.+\mu_{h} S_{H}^{*}-\left(\alpha A_{H}+\sigma I_{U}+\rho \psi I_{C}\right) S_{H}-\mu_{h} S_{H}\right) \\
& +\left(1-\frac{E_{H}^{*}}{E_{H}}\right)\left(\left(\alpha A_{H}+\sigma I_{U}+\rho \psi I_{C}\right) S_{H}\right. \\
& \left.-m_{1} E_{H}\right)+a\left(1-\frac{A_{H}^{*}}{A_{H}}\right)\left(\theta \beta E_{H}-m_{2} A_{U}\right) \\
& +b\left(1-\frac{I_{U}^{*}}{I_{U}}\right)\left(\omega(1-\theta) \beta E_{H}-m_{3} I_{U}\right) \\
& +c\left(1-\frac{I_{C}^{*}}{I_{C}}\right)\left((1-\omega)(1-\theta) \beta E_{H}-m_{4} I_{C}\right) .
\end{aligned}
$$

If we simplify further, we have

$$
\begin{aligned}
\frac{\mathrm{d} V}{\mathrm{~d} t} & =\left(\alpha A_{H}^{*}+\sigma I_{U}^{*}+\rho \psi I_{C}^{*}\right) S_{H}^{*}+m_{1} E_{H}^{*} \\
& +a m_{2} A_{H}^{*}+b m_{3} I_{U}^{*}+c m_{4} I_{C}^{*} \\
& -\left(\alpha A_{H}^{*}+\sigma I_{U}^{*}+\rho \psi I_{C}^{*}\right) \frac{\left(S_{H}^{*}\right)^{2}}{S_{H}} \\
& -\left(\alpha A_{H}+\sigma I_{U} \rho \psi I_{C}\right) \frac{S_{H} E_{H}^{*}}{E_{H}} \\
& -\frac{a \theta \beta E_{H} A_{H}^{*}}{A_{H}}-\frac{b \omega(1-\theta) \beta E_{H} I_{U}^{*}}{I_{U}} \\
& -\frac{c(1-\omega)(1-\theta) \beta E_{H} I_{C}^{*}}{I_{C}} \\
& +2 \mu_{h} S_{H}^{*}-\frac{\mu_{h}\left(S_{H}^{*}\right)^{2}}{S_{H}}-\mu_{h} S_{H} \\
& +\left(\alpha A_{H}+\sigma I_{U}+\rho \psi I_{C}\right) S_{H}^{*} \\
& -m_{1} E_{H}+a \theta \beta E_{H}-a m_{2} A_{H} \\
& +b \omega(1-\theta) \beta E_{H}-b m_{3} I_{U}-c m_{4} I_{C} \\
& +c(1-\omega)(1-\theta) \beta E_{H} .
\end{aligned}
$$

Substituting $a$ and $b$ by their values and exploiting 
(41) - (43) gives

$$
\begin{aligned}
a \theta \beta & =\frac{\alpha S_{H}^{*} A_{H}^{*}}{E_{H}^{*}} \\
b \omega(1-\theta) \beta & =\frac{\sigma S_{H}^{*} I_{U}^{*}}{E_{H}^{*}} \\
c(1-\omega)(1-\theta) \beta & =\frac{\rho \psi S_{H}^{*} I_{C}^{*}}{E_{H}^{*}} .
\end{aligned}
$$

Using (40) - (43) and (48) - (50), we have

$$
\begin{aligned}
\frac{\mathrm{d} V}{\mathrm{~d} t} & =\mu_{h} S_{H}^{*}\left(2-\frac{S_{H}^{*}}{S_{H}}-\frac{S_{H}}{S_{H}^{*}}\right) \\
& +3\left(\alpha A_{H}^{*}+\sigma I_{U}^{*}+\rho \psi I_{C}^{*}\right) S_{H}^{*} \\
& -\left(\alpha A_{H}^{*}+\sigma I_{U}^{*}+\rho \psi I_{C}^{*}\right) \frac{\left(S_{H}^{*}\right)^{2}}{S_{H}} \\
& -\left(\alpha A_{H}+\sigma I_{U}+\rho \psi I_{C}\right) \frac{S_{H} E_{H}^{*}}{E_{H}} \\
& -\frac{\alpha S_{H}^{*} E_{H}\left(A_{H}^{*}\right)^{2}}{E_{H}^{*} A_{H}}-\frac{\sigma S_{H}^{*} E_{H}\left(I_{U}^{*}\right)^{2}}{E_{H}^{*} I_{U}} \\
& -\frac{\rho \psi S_{H}^{*} E_{H}\left(I_{C}^{*}\right)^{2}}{E_{H}^{*} I_{C}}
\end{aligned}
$$

i.e.

$$
\begin{aligned}
\frac{\mathrm{d} V}{\mathrm{~d} t} & =\mu_{h} S_{H}^{*}\left(2-\frac{S_{H}^{*}}{S_{H}}-\frac{S_{H}}{S_{H}^{*}}\right) \\
+ & \alpha S_{H}^{*} A_{H}^{*}\left(3-\frac{S_{H}^{*}}{S_{H}}-\frac{S_{H} E_{H}^{*} A_{H}}{S_{H}^{*} E_{H} A_{H}^{*}}-\frac{\sigma E_{H} A_{H}^{*}}{E_{H}^{*} A_{H}}\right) \\
& +\sigma S_{H}^{*} I_{U}^{*}\left(3-\frac{S_{H}^{*}}{S_{H}}-\frac{S_{H} E_{H}^{*} I_{U}}{S_{H}^{*} E_{H} I_{U}^{*}}-\frac{E_{H} I_{U}^{*}}{E_{H}^{*} I_{U}}\right) \\
& +\left(3-\frac{S_{H}^{*}}{S_{H}}-\frac{S_{H} E_{H}^{*} I_{C}}{S_{H}^{*} E_{H} I_{C}^{*}}-\frac{E_{H} I_{C}^{*}}{E_{H}^{*} I_{C}}\right) .
\end{aligned}
$$

By arithmetic-geometric means inequality

$$
\frac{a_{1}+a_{2}+\cdots+a_{n}}{n} \geq \sqrt[n]{a_{1} a_{2} \cdots a_{n}}
$$

where $a_{1} a_{2} \cdots a_{n}=1$ and $a_{1}, a_{2}, \ldots, a_{n}>0$, i.e. $n-\left(a_{1}+a_{2}+\cdots+a_{n}\right) \leq 0$, it follows that $\frac{\mathrm{d} V}{\mathrm{~d} t} \leq 0$ with $V=0$ if and only if $S_{H}=S_{H}^{*}, E_{H}=E_{H}^{*}$, $A_{H}=A_{H}^{*}, I_{U}=I_{U}^{*}, I_{C}=I_{C}^{*}$.

Hence, the largest compact invariant subset of the set, where $\frac{\mathrm{d} V}{\mathrm{~d} t}=0$, is

$$
\left(S_{H}, E_{H}, A_{H}, I_{U}, I_{C}\right)=\left(S_{H}^{*}, E_{H}^{*}, A_{H}^{*}, I_{U}^{*}, I_{C}^{*}\right) .
$$

Therefore, by stability theorem of Lyapunov and LaSalle's Invariance Principle, it follows that every solution in $D$ approaches $E_{1}$ for $R_{c}>1$ as $t \rightarrow \infty$.

The epidemiological implication of the above result is that COVID-19 will institute itself in the population for $R_{c}>1$.

\section{Sensitivity Analysis}

We perform a sensitivity analysis of COVID-19 model to determine the parameters which has greater impact in the transmission dynamics and spread of the disease. Sensitivity analysis is helpful for experimental design, data assimilation and reduction of complex nonlinear models. A very high sensitivity indicates that more care should be taken in the estimation of the associated parameters. Following the approach by [30], we define sensitivity index of a variable to a parameter as the ratio of relative change in the variable to the relative change in the parameter.

When the variable is a differentiable function of any parameter, the sensitivity index may be alternatively defined using partial derivatives.

Definition 4.1: The normalized forward sensitivity index of $u$, that depends differentiably on a parameter $p$, is defined as

$$
N_{p}^{u}=\frac{\partial u}{\partial p} \times \frac{p}{u}
$$

for $u \neq 0$.

Consequently, we derive analytical expression for the sensitivity index of $R_{c}$ as

$$
N_{p_{i}}^{R_{c}}=\frac{\partial R_{c}}{\partial p_{i}} \times \frac{p_{i}}{R_{c}}
$$

where $p_{i}, i \in \mathbb{N}$ denotes each parameter involved in $R_{c}$.

$R_{c}$ is defined as

$$
R_{c}=\sqrt{\begin{array}{l}
\frac{S_{0} \beta \alpha \theta}{m_{1} m_{2}}+\frac{S_{0} \beta \sigma \omega(1-\theta)}{m_{1} m_{3}} \\
+\frac{S_{0} \beta \rho \psi(1-\omega)(1-\theta)}{m_{1} m_{4}}
\end{array}},
$$

where

$$
\begin{aligned}
& m_{1}=\beta+\mu_{h}, \\
& m_{2}=\kappa_{1}+\tau_{1}+\mu_{h}, \\
& m_{3}=\kappa_{2}+\delta_{1}+\tau_{2}+\mu_{h}, \\
& m_{4}=\kappa_{3}+\delta_{2}+\tau_{3}+\mu_{h} .
\end{aligned}
$$

Table 2 summarizes the sensitivity indices on $R_{c}$ with respect to parameters, i.e

$$
\begin{array}{r}
N_{\varepsilon}^{R_{c}}, N_{\alpha}^{R_{c}}, N_{\beta}^{R_{c}}, N_{\theta}^{R_{c}}, N_{\sigma}^{R_{c}}, N_{\omega}^{R_{c}}, N_{\rho}^{R_{c}}, N_{\psi}^{R_{c}}, N_{\mu_{h}}^{R_{c}}, \\
N_{\kappa_{1}}^{R_{c}}, N_{\kappa_{2}}^{R_{c}}, N_{\tau_{1}}^{R_{c}}, N_{\tau_{2}}^{R_{c}}, N_{\delta_{1}}^{R_{c}}, N_{\kappa_{3}}^{R_{c}}, N_{\tau_{3}}^{R_{c}}, N_{\delta_{2}}^{R_{c}} .
\end{array}
$$


Table 2: Numerical values of sensitivity indices of $R_{c}$ with respect to parameter involved

\begin{tabular}{|c||c|}
\hline \hline Parameter symbol & Sensitivity Index \\
\hline \hline$\varepsilon$ & +0.5000000 \\
\hline$\alpha$ & +0.4299384 \\
\hline$\beta$ & +0.1478873 \\
\hline$\theta$ & +0.0968840 \\
\hline$\sigma$ & +0.0700478 \\
\hline$\omega$ & +0.0699777 \\
\hline$\rho$ & +0.0000137 \\
\hline$\psi$ & +0.0000137 \\
\hline$\mu_{h}$ & -0.6952639 \\
\hline$\kappa_{1}$ & -0.3430359 \\
\hline$\kappa_{2}$ & -0.0517594 \\
\hline$\tau_{1}$ & -0.0457381 \\
\hline$\tau_{2}$ & -0.0069012 \\
\hline$\delta_{1}$ & -0.0051759 \\
\hline$\kappa_{3}$ & -0.0000101 \\
\hline$\tau_{3}$ & -0.0000013 \\
\hline$\delta_{2}$ & -0.0000010 \\
\hline
\end{tabular}

\subsection{Interpretation of sensitivity indices obtained in Table 2}

It can be deduced from the results for sensitivity analysis that $R_{c}$ is most sensitive to the parameter $\varepsilon$, followed by $\alpha$ and $\beta$ in a positive sense. $R_{c}$ is also most sensitive to the parameter $\mu_{h}$, followed by $\kappa_{1}$ and $\kappa_{2}$ (hospitalization rate of the unconcerned and concerned infectious individuals) in a negative sense. Other parameters that strongly influence the dynamics of COVID-19 infection are $\theta, \sigma, \omega, \rho, \psi, \tau_{1}, \tau_{2}, \delta_{1}, \kappa_{3}, \tau_{3}, \delta_{2}$. Parameters $\theta, \sigma, \omega, \rho$ and $\psi$ have a positive influence on the basic reproduction number $R_{c}$, that is an increase in these parameters implies an increase in $R_{c}$. Parameters $\tau_{1}, \tau_{2}, \delta_{1}, \kappa_{3}, \tau_{3}$ and $\delta_{2}$ have a negative influence on the basic reproduction number, that is an increase in these parameters implies a decrease in $R_{c}$. Consequently, the sensitivity indices for the model are graphically shown below. As a result of the sensitivity analysis, the following suggestions are made:

(1) Proper quarantining of the incoming travellers could reduce the value of $\varepsilon$.

(2) An increase in a compulsory massive testing of the exposed individuals may help in minimizing the size of the asymptomatic, unconcerned and concerned individuals by reducing the value of $\beta, \theta, \omega$.

(3) Hospitalizing the asymptomatic infectious and unconcerned infectious individuals after compulsory massive testing, is another good control measure against COVID-19 infection because it helps to re- duce the value of transmission rate $\alpha$ and $\sigma$.

(4) Educating the populace of the danger of noncompliance to COVID-19 preventive measures could reduce the size of the unconcerned infectious individuals.

\section{Numerical Simulations}

Here, we carried out a numerical simulation of the asymptomatic behaviour of COVID-19 model (1) (7) using MATLAB software package. The simulation of the dynamic behaviour of the susceptible human population is shown in Fig.1(a) while the simulation of the dynamic behaviour of the exposed human population is shown in Fig.1(b). Simulation showing the dynamic behaviour of the asymptomatic infected and unconcerned infected human population were displayed in Fig.2(a) and 2(b), respectively. It was observed that the simulation showing the dynamic behaviour of the concerned infected human, hospitalised infected and recovered human population were also displayed in Fig.3(a), 3(b), and 3(c) for different initial conditions, so that $R_{c}<1$. We noticed that in Fig. $1-9$ that there were variations in the parameter values for the contact rates between susceptible and unconcerned infectious $\sigma$. In Fig. $1-3$ the parameter values for $\sigma=0.001$ which produces the result obtained in the simulations. It was observed from Fig.1(a) that the susceptible human population continue to increase until they reached a state where they maintained a steady state after a certain period of time while Fig. 1(b) shows that the number of exposed human depletes after a short while and all the exposed goes into extinction. We also observed the same extinction for the asymptomatic and unconcerned infected human population in Fig.2(a) and 2(b), respectively. The populations of the concerned infected, hospitalized and recovered human population also decreases over time in Fig.3(a), 3(b), and 3(c), respectively, given that the value of the contact rate between susceptible and unconcerned infected is assumed to be very low. Thus, the solution trajectories converge to the disease-free equilibrium, showing that the disease-free equilibrium solution is globally asymptotically stable. This means that population that starts with COVID-19 infection shrinks and never turns to epidemic for $R_{c}<1$. When the contact rate between the susceptible and unconcerned infected $\sigma$ increases, in Fig.4(a) and 4(b) it was observed that the susceptible population increases for a short period of time and then depleted into a steady state over a long period of time, while the exposed human population increases and then dropped slightly to maintain a steady state over a long period of time. It was observed that the asymptomatic infectious population dropped and then increases to maintain a steady state over a long period of time as shown in Fig.5(a), and the popu- 
lation of the unconcerned infected also dropped and then increases slightly to maintain a steady state in Fig.5(b). In Fig.6(a) we observed that the population of the unconcerned infected human dropped and then increases to maintain a steady state over a long period of time. The population of the hospitalized infected increases and then dropped slightly to maintain a steady state over a long period of time. In Fig.6(b) it was observed that the recovered human population increases and then maintained a steady state over a long period of time. It can be shown that, irrespective of the initial condition, the infected population (the exposed, the asymptomatic infectious, the unconcerned and concerned infectious individuals) remains in the population and stabilizes in time. This means that the solution trajectories converge to the endemic equilibrium solution. Therefore, COVID-19 infection institutes itself in the population for $R_{c}>1$, as established in Theorem 3.2. When $\sigma$ is assumed to be increased much more, it was observed that the susceptible population dropped sporadically, increases slightly and then dropped to maintain a steady state over a long period of time in Fig.7(a). We observed in Fig.7(b) that the exposed human population increases over time to maintain a steady state. In Fig.8(a) we also noticed that the asymptomatic infected population dropped and then increases to maintain a steady state over a long period of time. The population of the unconcerned infected human also dropped and then increased to maintain a steady state over a long period of time. Fig.9(a) showed that the concerned infected population dropped and then increases to maintain a steady state over a long period of time. In Fig.9(b) the population of the hospitalized infected increases over time to maintain a steady state over a long period of time. In Fig.9(b) we also observed that the population of the recovered human increases to maintain a steady state over a long period of time. it can be shown that, irrespective of the initial condition, the infected population (the exposed, the asymptomatic infectious, the unconcerned and concerned infectious individuals) remains in the population and stabilizes in time. This means that the solution trajectories converge to the endemic equilibrium solution. Therefore, COVID-19 infection institutes itself in the population for $R_{c}>1$, as established in Theorem 3.2.

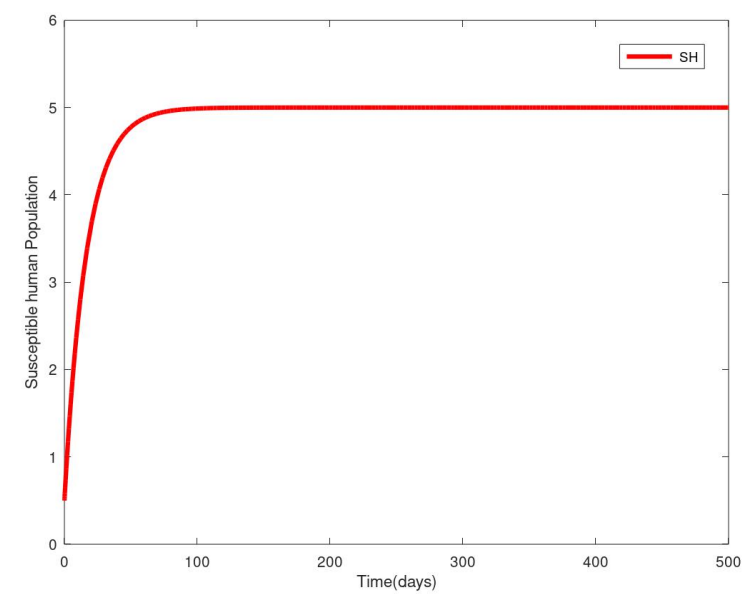

(a)

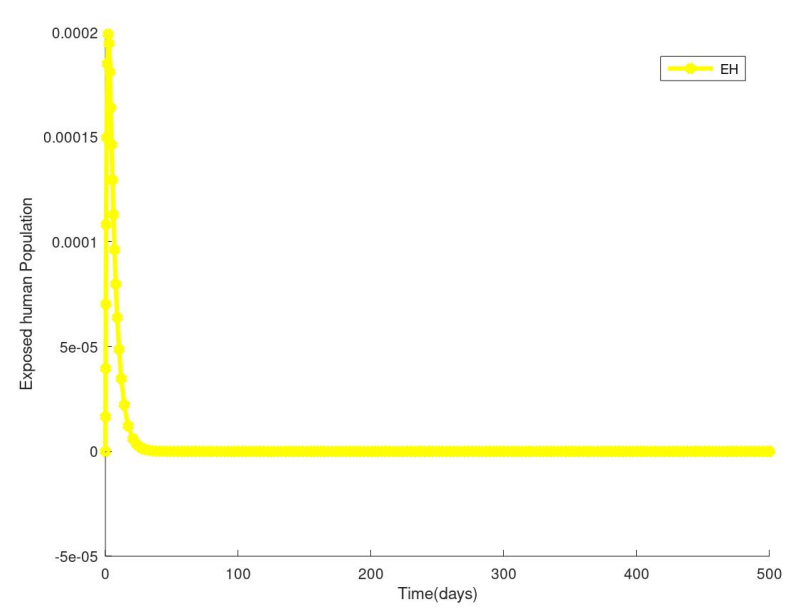

(b)

Figure 1: Simulation showing the dynamic behaviour of the susceptible human and exposed human population and is given for $\beta_{1}=0.2143, \alpha=0.001$, $\rho=0.001, \sigma=0.001, \theta=0.8262, \omega=0.8262$, $\tau_{1}=\tau_{2}=\tau_{3}=\tau_{4}=0.0667, \beta=0.142, \varepsilon=0.3$, $\mu_{h}=0.06, \delta_{1}=\delta_{2}=\delta_{3}=0.1, \kappa_{1}=\kappa_{2}=\kappa_{3}=1$, $\psi=1$. 


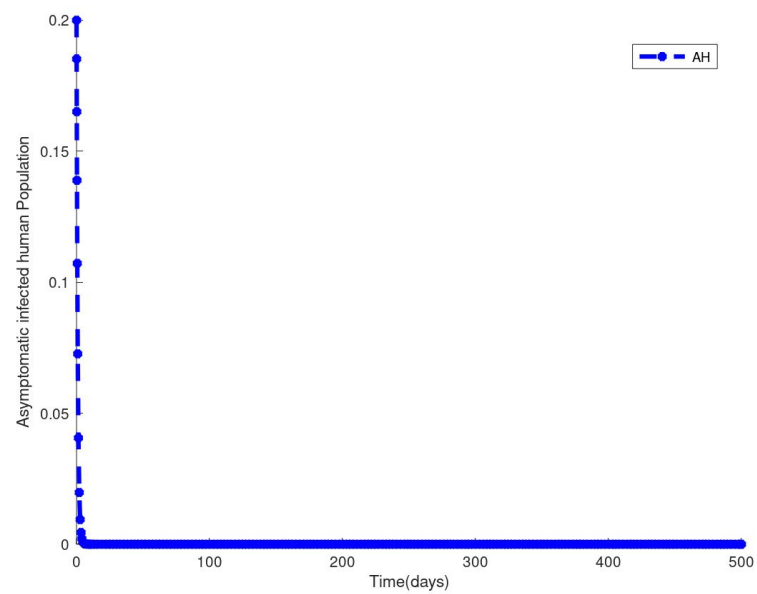

(a)

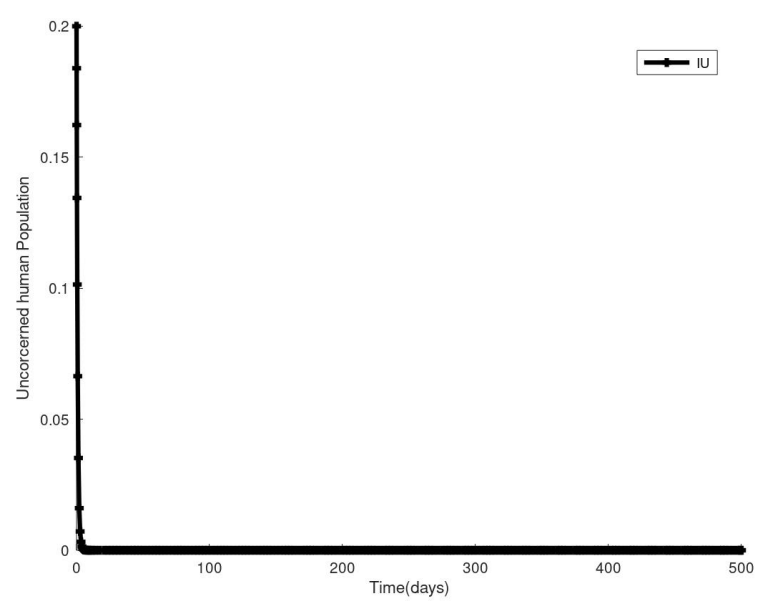

(b)

Figure 2: Simulation showing the dynamic behaviour of the asymptomatic infected and unconcerned infected human population and is given for $\beta_{1}=$ 0.2143, $\alpha=0.001, \rho=0.001, \sigma=0.001, \theta=$ $0.8262, \omega=0.8262, \tau_{1}=\tau_{2}=\tau_{3}=\tau_{4}=0.0667$, $\beta=0.142, \varepsilon=0.3 \mu_{h}=0.06, \delta_{1}=\delta_{2}=\delta_{3}=0.1$, $\kappa_{1}=\kappa_{2}=\kappa_{3}=1, \psi=1$.

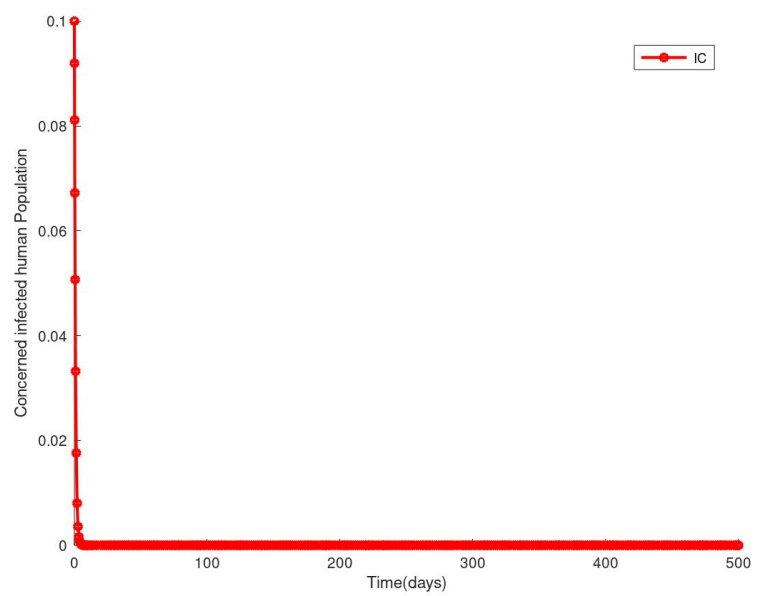

(a)

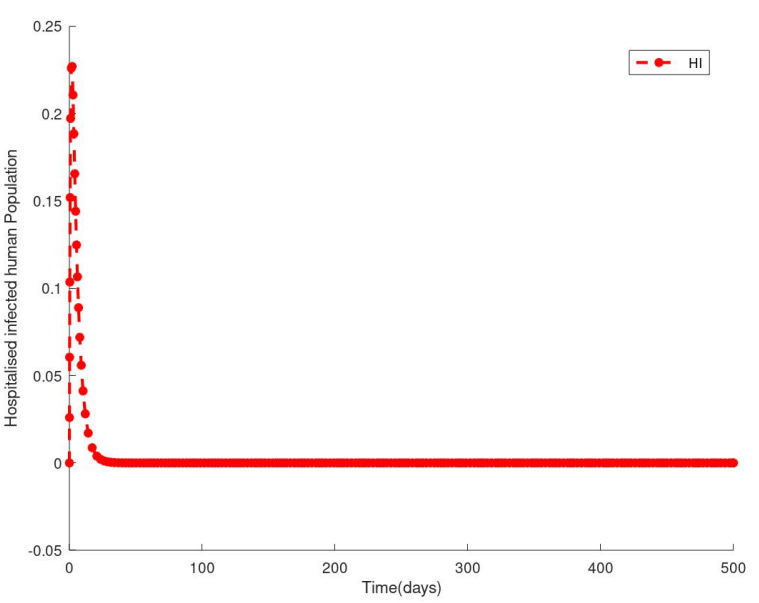

(b)

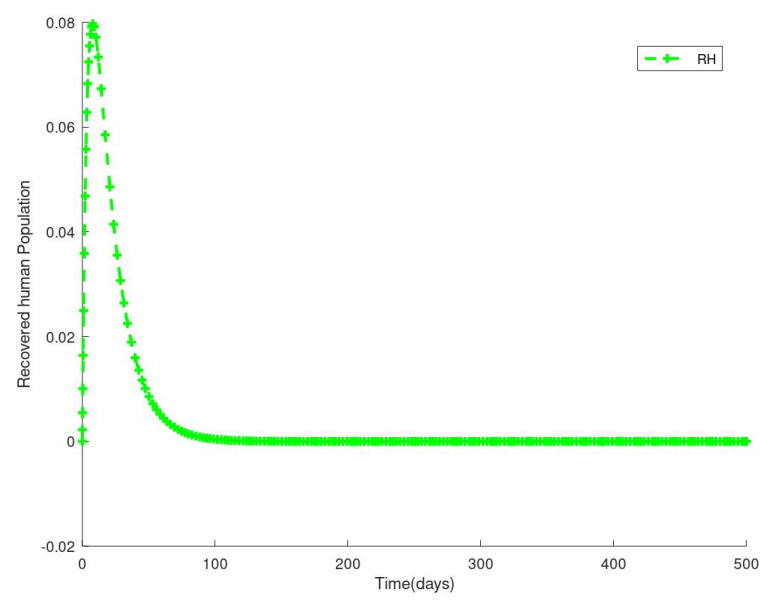

(c)

Figure 3: Simulation showing the dynamic behaviour of the concerned infected human, hospitalised human population and recovered human population and is given for $\beta_{1}=0.2143, \alpha=0.001, \rho=0.001$, $\sigma=0.001, \theta=0.8262, \omega=0.8262, \tau_{1}=\tau_{2}=$ $\tau_{3}=\tau_{4}=0.0667, \beta=0.142, \varepsilon=0.3, \mu_{h}=0.06$, $\delta_{1}=\delta_{2}=\delta_{3}=0.1, \kappa_{1}=\kappa_{2}=\kappa_{3}=1, \psi=1$. 


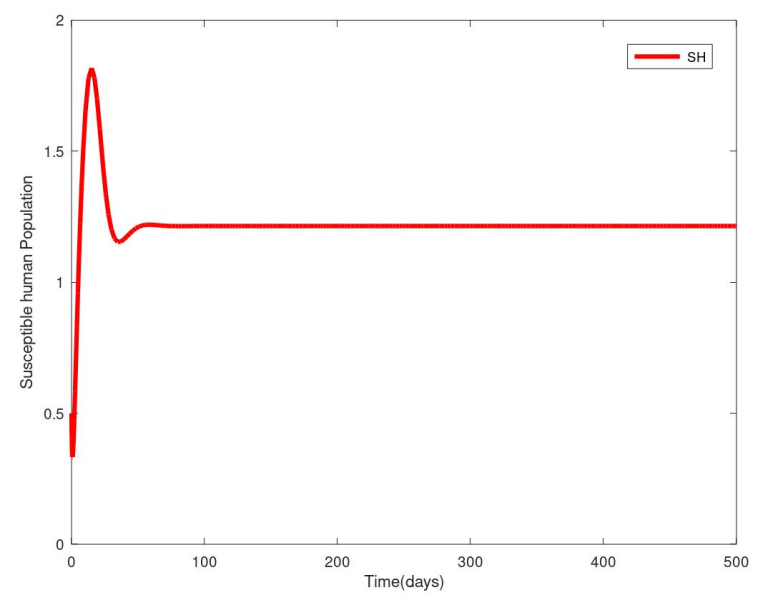

(a)

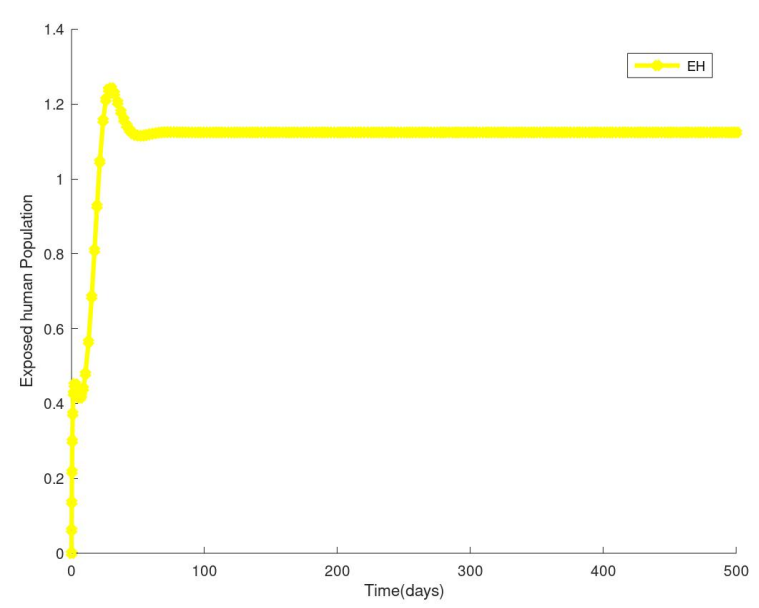

(b)

Figure 4: Simulation showing the dynamic behaviour of the susceptible human and exposed human population and is given for $\beta_{1}=0.2143, \alpha=0.001$, $\rho=0.001, \sigma=10, \theta=0.8262, \omega=0.8262$, $\tau_{1}=\tau_{2}=\tau_{3}=\tau_{4}=0.0667, \beta=0.142, \varepsilon=0.3$, $\mu_{h}=0.06, \delta_{1}=\delta_{2}=\delta_{3}=0.1, \kappa_{1}=\kappa_{2}=\kappa_{3}=1$, $\psi=1$.

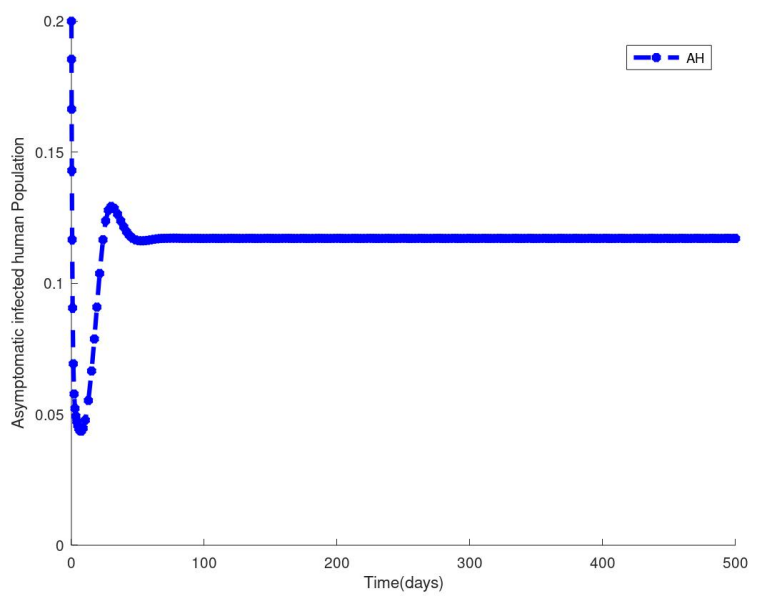

(a)

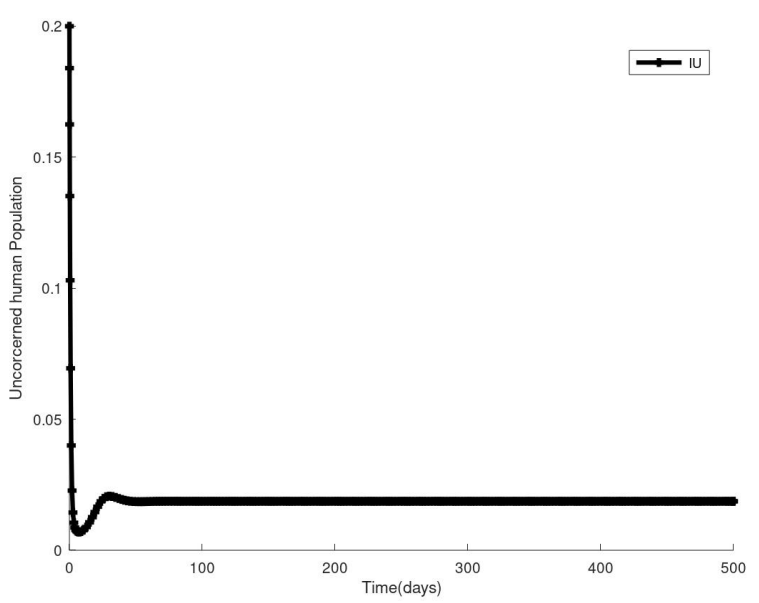

(b)

Figure 5: Simulation showing the dynamic behaviour of the asymptomatic infected and unconcerned infected human population and is given for $\beta_{1}=$ $0.2143, \alpha=0.001, \rho=0.001, \sigma=10, \theta=0.8262$, $\omega=0.8262, \tau_{1}=\tau_{2}=\tau_{3}=\tau_{4}=0.0667$, $\beta=0.142, \varepsilon=0.3, \mu_{h}=0.06, \delta_{1}=\delta_{2}=\delta_{3}=0.1$, $\kappa_{1}=\kappa_{2}=\kappa_{3}=1, \psi=1$. 


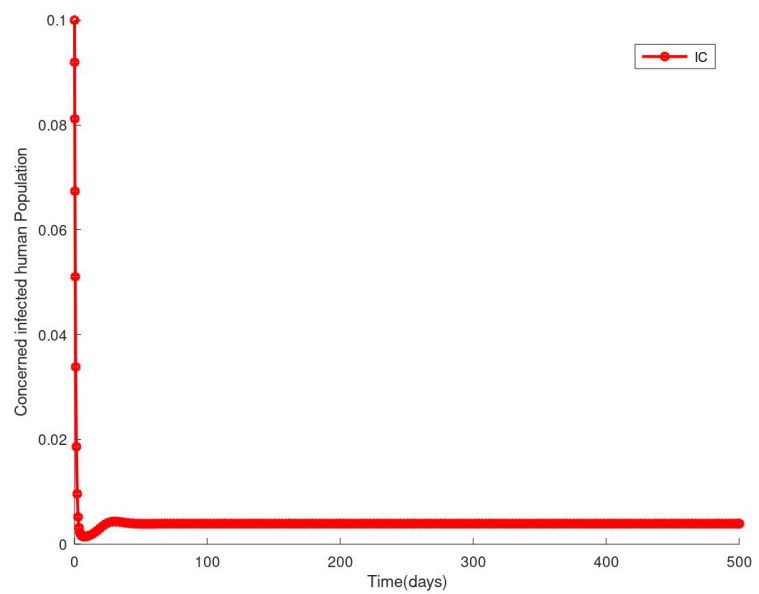

(a)

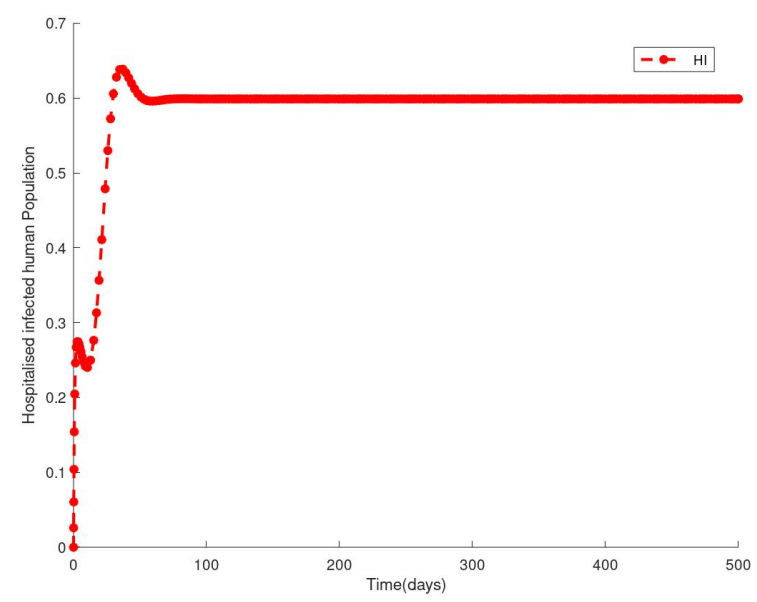

(b)

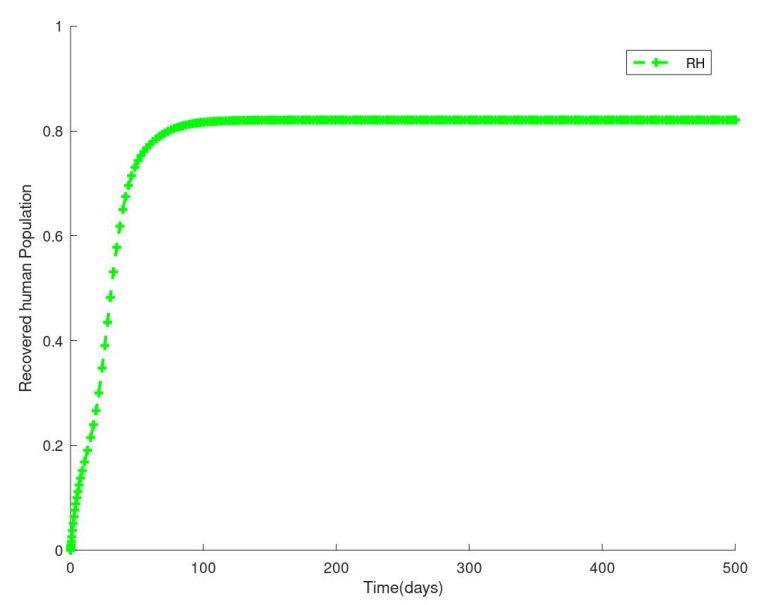

(c)

Figure 6: Simulation showing the dynamic behaviour of the concerned infected human, hospitalised human population and recovered human population and is given for $\beta_{1}=0.2143, \alpha=0.001, \rho=0.001$, $\sigma=10, \theta=0.8262, \omega=0.8262, \tau_{1}=\tau_{2}=$ $\tau_{3}=\tau_{4}=0.0667, \beta=0.142, \varepsilon=0.3, \mu_{h}=0.06$, $\delta_{1}=\delta_{2}=\delta_{3}=0.1, \kappa_{1}=\kappa_{2}=\kappa_{3}=1, \psi=1$.

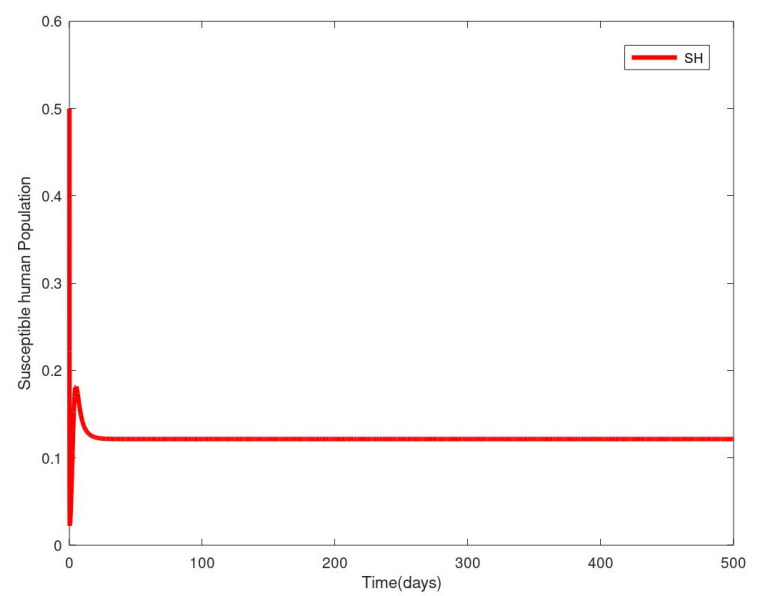

(a)

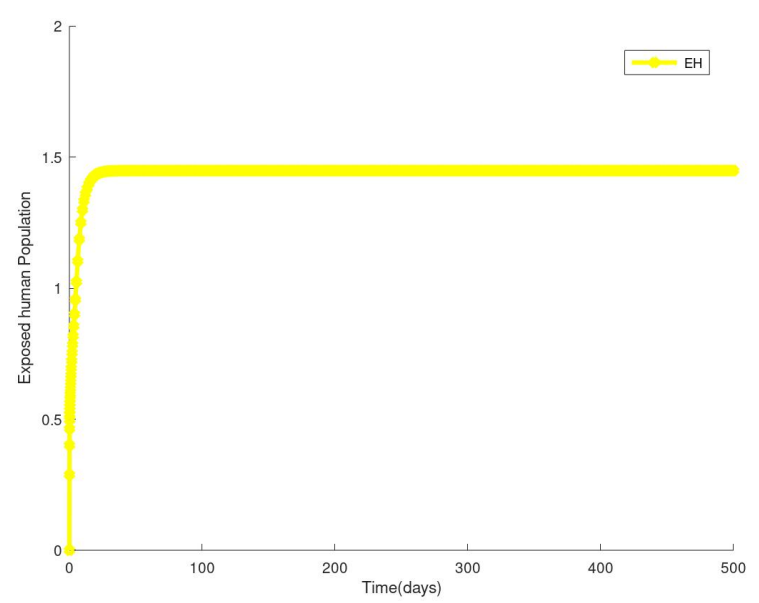

(b)

Figure 7: Simulation showing the dynamic behaviour of the susceptible human and exposed human population and is given for $\beta_{1}=0.2143, \alpha=0.001$, $\rho=0.001, \sigma=100, \theta=0.8262, \omega=0.8262$, $\tau_{1}=\tau_{2}=\tau_{3}=\tau_{4}=0.0667, \beta=0.142, \varepsilon=0.3$, $\mu_{h}=0.06, \delta_{1}=\delta_{2}=\delta_{3}=0.1, \kappa_{1}=\kappa_{2}=\kappa_{3}=1$, $\psi=1$. 


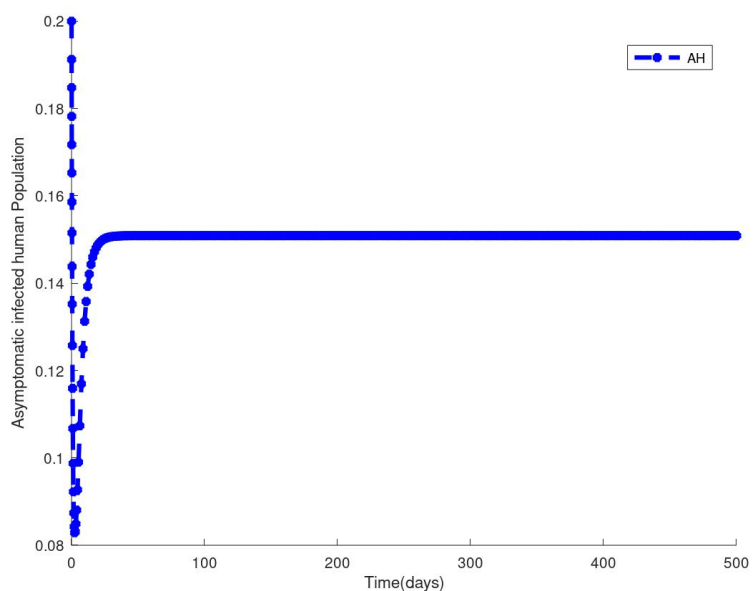

(a)

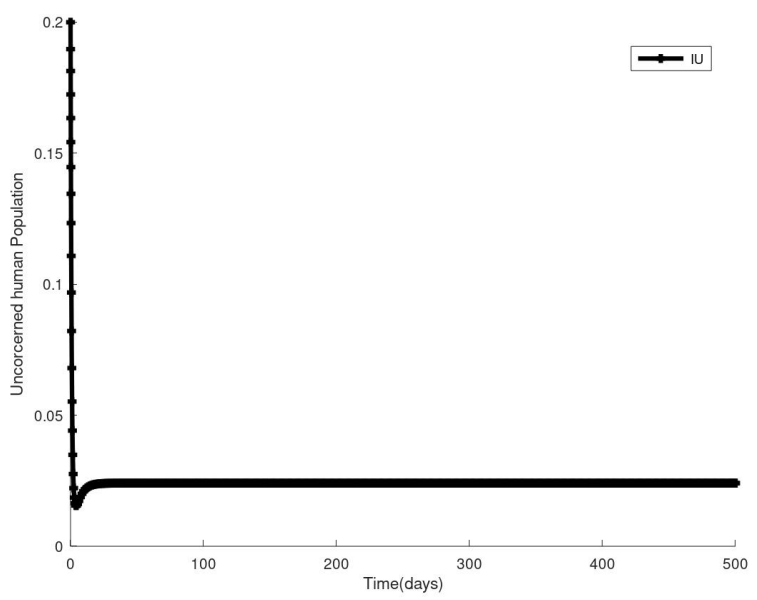

(b)

Figure 8: Simulation showing the dynamic behaviour of the asymptomatic infected and unconcerned infected human population and is given for $\beta_{1}=$ $0.2143, \alpha=0.001, \rho=0.001, \sigma=100, \theta=0.8262$, $\omega=0.8262, \tau_{1}=\tau_{2}=\tau_{3}=\tau_{4}=0.0667$, $\beta=0.142, \varepsilon=0.3, \mu_{h}=0.06, \delta_{1}=\delta_{2}=\delta_{3}=0.1$, $\kappa_{1}=\kappa_{2}=\kappa_{3}=1, \psi=1$.

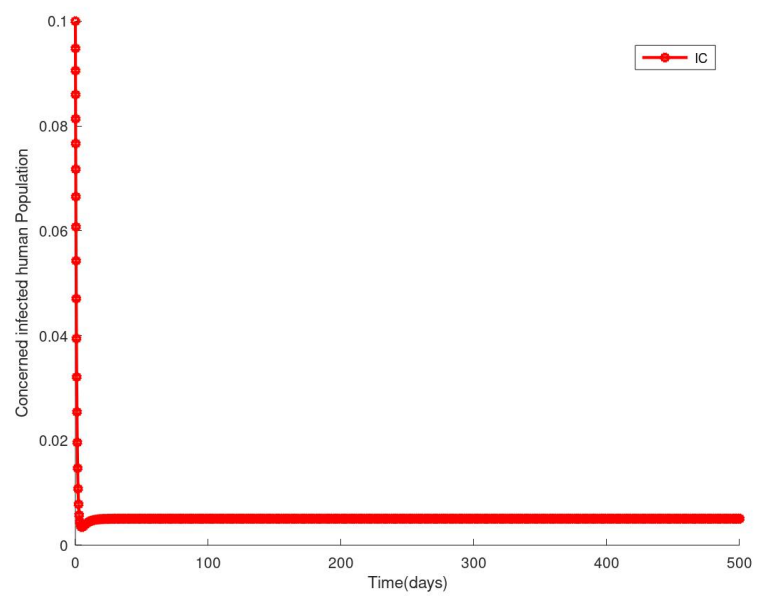

(a)

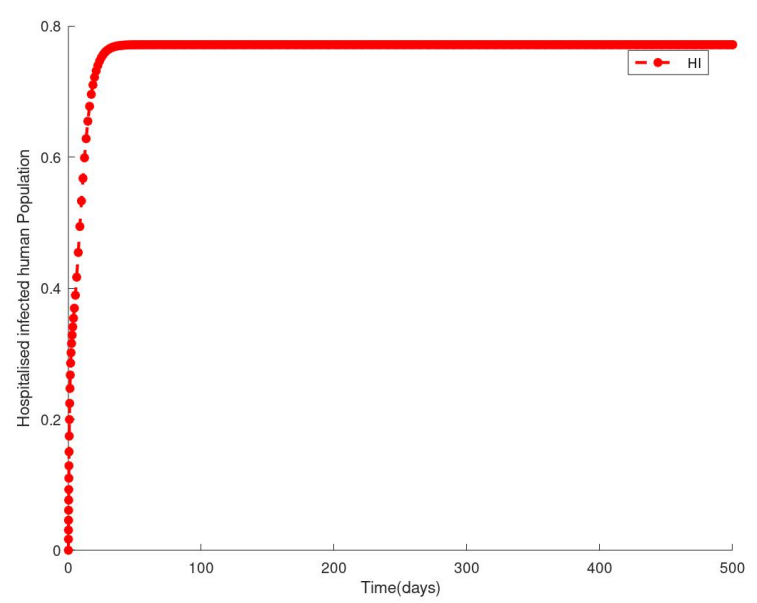

(b)

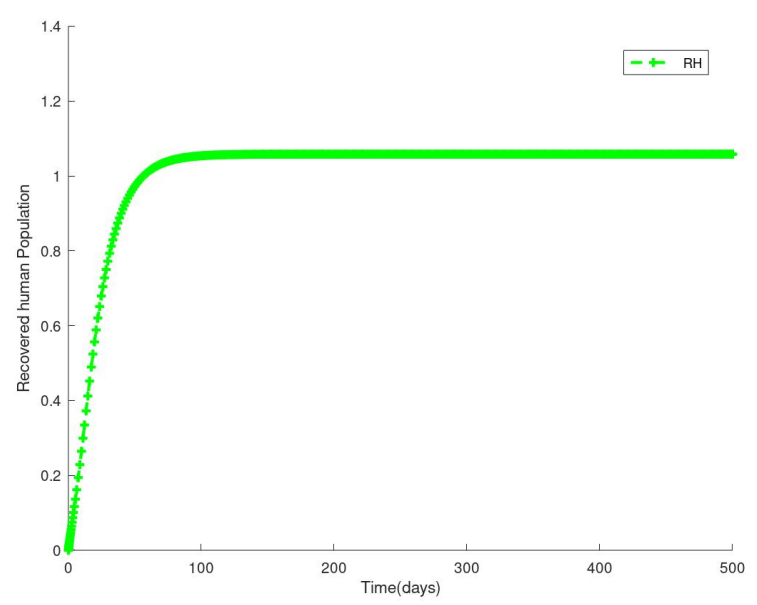

(c)

Figure 9: Simulation showing the dynamic behaviour of the concerned infected human, hospitalised human population and recovered human population and is given for $\beta_{1}=0.2143, \alpha=0.001, \rho=0.001$, $\sigma=100, \theta=0.8262, \omega=0.8262, \tau_{1}=\tau_{2}=$ $\tau_{3}=\tau_{4}=0.0667, \beta=0.142, \varepsilon=0.3, \mu_{h}=0.06$, $\delta_{1}=\delta_{2}=\delta_{3}=0.1, \kappa_{1}=\kappa_{2}=\kappa_{3}=1, \psi=1$. 


\section{Discussion and Conclusion}

We extended a basic COVID-19 model (susceptible, exposed, asymptomatic infectious, infectious individuals, hospitalized individuals, and recovered individuals) to a model that stratifies the infectious population to unconcerned and concerned infectious individuals, in which we focus more on the unconcerned infectious population. The amplitude of this group (the unconcerned infectious individuals) is higher than the amplitude of the concerned infectious compartment as shown in the numerical simulations for $R_{c}>1$. The motivation was the need to investigate the contribution of unconcerned infectious individuals on the reported resurgence and prevalence of the disease in Nigeria. The disease-free and endemic equilibria of the model were obtained and their global stability analyses were established. Precisely, we proved that the disease-free equilibrium point is globally asymptotically stable if $R_{c}<1$ and unstable if $R_{c}>1$. Sensitivity analysis of the basic reproduction number $R_{c}$ was also carried out and it revealed that the important parameters are the recruitment rate, contact rate, and hospitalization rate of the asymptomatic infectious and unconcerned infectious individuals. $\mathrm{Nu}$ merical simulations are carried out to show that the infected population (the exposed, the asymptomatic infectious, the unconcerned and the concerned infectious individuals) shrinks with time when $R_{c}<1$, whenever the value of the contact rate between susceptible and unconcerned infectious human is assumed to be very low (see Fig.1 - 3) but persists in the population when $R_{c}>1$ (see Fig.4-9), whenever the value of the contact rate between susceptible and unconcerned infectious human is assumed to be high or very high.

Looking at the situation in Nigeria, after the asymptomatic infectious group, hospitalizing the unconcerned infectious individuals, after compulsory massive testing is paramount in halting the transmission of COVID-19 infection. Also, attempts should be made to improve on the COVID-19 drug for fast recovery of the infectious population (asymptomatic infectious $\left(\tau_{1}\right)$ and unconcerned infectious $\left(\tau_{2}\right)$ individuals). Based on the results of the analyses, the following recommendations are listed below to halt the spread of COVID-19 infection in the population:

(1) Enhancing continuously, awareness through effective risk communication concerning COVID-19 to the general public, to reduce the number of unconcerned infectious individuals in the population.

(2) Strict quarantining of returning travellers from affected areas.

(3) Hospitalizing the infected population as a result of compulsory national testing.

(4) Scientific research efforts should be intensified in developing an effective COVID-19 drug for immediate treatment of any COVID-19 infected patient.

(5) Total compliance to all the COVID-19 preventive measures should be practised by every individual. These include making wearing a mask a normal part of being around other people, maintaining at least a 1-metre distance between an individual and others to reduce the risk of infection when they cough, sneeze or speak, avoiding crowded or indoor settings, regular hand washing before and after wearing face masks etc.

\section{Area of Further Development}

Our model can be extended by formulating its stochastic version in order to investigate the possibility of randomness in the spread of COVID-19 in the population.

Conflict of Interest: The authors declare no conflict of interest.

\section{References:}

[1] Chen, W., Peter, W. H., Frederick, G. H., George, F. G. A novel coronavirus of global health concern, The Lancet, 395, 470-473, (2020)

[2] World Health Organization, Coronavirus disease (COVID-19) Weekly Epidemiological Update and Weekly Operational Update, 2020, (2020). https://www.who.int/emergencies/diseases/

[3] Ken, L. L., Wang, Z. M., Wu, Z. Q., Xiang, Z. C., Guo, L., Xu, T., et al. Identification of a novel coronavirus causing severe pneumonia in human, a descriptive study, Chinese Med. J., (2020). https://doi.org/10.1097/CM9.00000000000007

[4] Anderson, R. M., Heesterbeek, H., Klinkenberg, D., Hollingsworth, D. How will country-based mitigation measures influence the course of the COVID-19 epidemic?, The Lancet, (2020). https://doi.org/10.1016/S0140-6736(20)305675

[5] Biao, T., Luigi Bragazzi, N., Li, Q., Tang, S., Xiao, Y., Wu, J. An updated estimation of the risk of transmission of the novel coronavirus (2019-nCov), Infectious Disease Modeling, 5, 248-255, (2020)

[6] Adam, J. K., Timothy, W. R., Charlie, D., Yang, L., John, E., Sebastian, F., Rosalind, M. E. Early dynamics and transmission and control of COVID-19: a mathematical modeling study, Center for Mathematical Modeling of Infectious Diseases, (2020) 
[7] Ying, L., Gayle, A. A., Wilder-Smith, A., Rocklöw, J. The reproduction number of COVID-19 is higher compared to SARS coronavirus, Journal of Travel Medicine, 1-4, (2020)

[8] Hernandez-Vargas, E. A., VelascoHernandez, J. X. In host modeling of COVID-19 in Humans, (2020). https://doi.org/10.1101/2020.03.26.20044487

[9] Ivorra, B., Ferrandez, M. R., Vela-Perez M., Ramos, A. M. Mathematical modeling of the spread of the coronavirus disease 2019 (COVID-19) considering its particular characteristics. The case of China, (2020). https://doi.org/10.1016/j.cnsns.2020.105303

[10] Tridip, S., Shahid Nadim, S. K., Sourav, R., Joydev, C. Assessment of 21 days lockdown effect in some states and overall India: A predictive mathematical study on COVID-19 outbreak, Chaos, Solitions and Fractals, 139, (2020)

[11] Nkague Nkamba, L., Mann Manyombe, M. L., Manga, T. T., Mbang, J. Modeling Analysis of a SEIQR Epidemic Model to Assess the Impact of Undetected Cases and Containment Measures of the COVID-19 Outbreak in Cameroon, London Journal Press, 20 (2020)

[12] Enahoro, A. I., Sharomi, O. O., Ngonghala, C. N., Gumel, A. B. Mathematical modeling and analysis of COVID-19 in Nigeria, Mathematical Biosciences and Engineering, 17(6), 7192-7220 (2020)

[13] Alsaeed, N. I., Alqaissi, E. Y., Siddiqui, M. A. An Agent-based Simulation of the SIRD model of COVID-19 Spread, International Journal of Biology and Biomedical Engineering, 14, 210-217 (2020)

[14] Rattanakul, C., Lenbury, Y. Model Analysis and Simulation on Impacts of COVID-19 Pandemic on the Economy: a Case Study of Thailand's Gdp and its Lock Down Measures, International Journal of Biology and Biomedical Engineering, 14, 180-190 (2020)

[15] Olaniyi, S., Obabiyi, O. S., Okosun, K. O., Oladipo, A. T., Adewale, S. O. Mathematical Modeling and Optimal Cost-Effectiveness Control of COVID-19 Transmission Dynamics, The European Physical Journal Plus 135(11): 938, (2020)

[16] Adeniyi, M. O., Ekun, M. I., Iluno, C., Ogunsanya, A. S., Akinyemi, J. A., Oke, S. I.,
Matadi, M. B. Dynamic model of COVID-19 disease with exploratory data analysis, Scientific African, Elsevier, (2020)

[17] Enahoro, A. I., Ngonghala, C. N., Gumel, A. B. Will an imperfect vaccine curtail the COVID-19 pandemic in the US?, Infectious Disease Modeling, 5, 510-524, (2020)

[18] Adamoniene, R. Management Presumptions and Possibilities of Human Resources Formation, Bekesiene S., Hoskova-Mayerova S. (eds.), Challenges to National Defence in Contemporary Geopolitical Situation (CNDCGS' 2018), 157-166, (2018)

[19] Švarcová, I., Ptáček, B., Navrátil, J. Psychological intervention as support in disaster preparedness, In: Crisis Management and Solution of the Crisis Situations 2015, 317-320, (2015)

[20] Tušer, I. The development of education in emergency management, Studies in Systems, Decision and Control 247, 169-175, (2020). https://doi.org/10.1007/978-3-030-30659-5-10

[21] Tušer, I., Navrátil, J. Evaluation Criteria of Preparedness for Emergency Events within the Emergency Medical Services, Studies in Systems, Decision and Control 208, 463472 (2020). https://doi.org/10.1007/978-3-03018593-0-33

[22] Urban, R., Hoskova-Mayerova, S. Threat life cycle and its dynamics. Deturope 9 (2), 93-109, (2017)

[23] Lasalle, J. P. The stability of dynamical systems, SIAM Rev., 21(3), 418-420, (1976)

[24] Pien, H. What we know about the silent spreaders of COVID-19. The coronavirus crisis, WABE.org, (2020)

[25] Read, J. M., Bridgen, J. R. E., Cummings, D. A. T., et al. Novel coronavirus 2019-nCoV: early estimation of epidemiological parameters and epidemic predictions, MedRxiv, (2020). https://www.medrxiv.org/content/10.1101/2020

[26] Tang, B., Wang, X., Li, Q., Bragazzi, N. L., Tang, S., Xiao, Y., Wu, J. Estimation of the transmission risk of the 2019-nCoV and its implication for public health intervensions, Journal of Clinical Medicine, 9(2): 462, (2020)

[27] Lauer, S. A., Grantz, K. H., Bi, Q., Jones, F. K., et al. The incubation period of coronavirus disease 2019 (COVID-19) from publicly reported confirmed cases: Estimation and 
Application, Annals of Internal Medicine, 172, 577-582, (2020)

[28] Ferguson, N. M., Laydon, D., Nedjati-Gilani, G., et al. Impact of non-pharmaceutical interventions (NPIS) to reduce COVID-19 mortality and healthcare demand, Imperial College, London, (2020)

[29] Guo, H., Li, M.Y. Global stability in a mathematical model of tuberculosis, Canadian Applied Mathematics quaterly, 14(2), (2006)

[30] Arriola, L. M., Hyman, J. M. Being sensitive to uncertainty, Journal of Computing and Engineering, (2007)

\section{Contribution of individual authors to} the creation of a scientific article (ghostwriting policy)

T. S. Faniran carried out the modeling and the optimization.

E. A. Bakare provided the data, numerical simulations and their basic processing.

R. Potucek and E. O. Ayoola were responsible for the final editing of the text in the $\mathrm{BT}_{\mathrm{E}} \mathrm{X}$ typesetting system.

Follow:

https://www.wseas.org/multimedia/contributor-role

\section{Creative Commons Attribution License 4.0} (Attribution 4.0 International, CC BY 4.0)

This article is published under the terms of the Creative Commons Attribution License 4.0

https://creativecommons.org/licenses/by/4.0/deed.en US 